\title{
Reduced risk of venous thromboembolism with the use of intermittent pneumatic compression after craniotomy: a randomized controlled prospective study
}

\author{
Julian Prell, MD, ${ }^{1}$ Grit Schenk, MD, ${ }^{1}$ Bettina-Maria Taute, MD, ${ }^{2}$ Christian Scheller, MD, ${ }^{1}$ \\ Christian Marquart, MD, ${ }^{1}$ Christian Strauss, MD, ${ }^{1}$ and Stefan Rampp, MD ${ }^{1}$ \\ Departments of ${ }^{1}$ Neurosurgery and ${ }^{2}$ Angiology, University of Halle, Germany
}

OBJECTIVE The term "venous thromboembolism" (VTE) subsumes deep venous thrombosis (DVT) and pulmonary embolism. The incidence of DVT after craniotomy was reported to be as high as $50 \%$. Even clinically silent DVT may lead to potentially fatal pulmonary embolism. The risk of VTE is correlated with duration of surgery, and it appears likely that it develops during surgery. The present study aimed to evaluate intraoperative use of intermittent pneumatic compression (IPC) of the lower extremity for prevention of VTE in patients undergoing craniotomy.

METHODS A total of 108 patients undergoing elective craniotomy for intracranial pathology were included in a singlecenter controlled randomized prospective study. In the control group, conventional compression stockings were worn during surgery. In the treatment group, IPC of the calves was used in addition. The presence of DVT was evaluated by Doppler sonography pre- and postoperatively.

RESULTS Intraoperative use of IPC led to a significant reduction of VTE $(p=0.029)$. In logistic regression analysis, the risk of VTE was approximately quartered by the use of IPC. Duration of surgery was confirmed to be correlated with VTE incidence $(p<0.01)$; every hour of surgery increased the risk by a factor of 1.56 .

CONCLUSIONS Intraoperative use of IPC significantly lowers the incidence of potentially fatal VTE in patients undergoing craniotomy. The method is easy to use and carries no additional risks.

- CLASSIFICATION OF EVIDENCE Type of question: therapeutic; study design: randomized controlled trial; evidence: class I.

Clinical trial registration no.: DRKS00011783 (https://www.drks.de)

https://thejns.org/doi/abs/10.3171/2017.9.JNS17533

KEYWORDS venous thromboembolism; deep venous thrombosis; intermittent pneumatic compression; craniotomy

$\mathrm{V}$ ENOus thromboembolism (VTE) subsumes deep venous thrombosis (DVT) and pulmonary embolism (PE). After craniotomy, up to $50 \%$ of all patients will develop VTE. ${ }^{11,17}$ In the majority of cases, this condition is asymptomatic. Still, symptomatic VTE is seen in $7.5 \%$ of patients undergoing craniotomy, $1,7,37$ and even asymptomatic VTE localized in distal muscle veins may progress to symptomatic thrombosis of the deep venous system. ${ }^{22,25,26,31}$ This may lead-directly and without clinical warning-to PE in $7 \%$ of the affected patients. ${ }^{16}$ Such PE derives from DVT in $>90 \%$ of all cases,${ }^{10}$ is seen in $3.7 \%$ of all patients undergoing craniotomy, and is lethal in up to $50 \%$ of all affected neurosurgical patients..$^{17,23}$
These numbers show that VTE is frequent and that it may severely impair or even kill without warning patients who have been successfully treated for intracranial pathology. Consequently, its prevention is of paramount importance in clinical management of neurosurgical patients. Postoperative chemoprophylaxis with low-molecular-weight heparin (LMWH) combined with compression stockings is in widespread use ${ }^{30}$ However, the incidence of VTE has been measured to be $39.6 \%$ after craniotomy although these methods were used in a prospective study. ${ }^{29}$

The aforementioned study ${ }^{29}$ also demonstrated that duration of surgery was an independent risk factor for VTE after craniotomy. Based on these findings, it was hypoth-

ABBREVIATIONS DVT = deep venous thrombosis; IPC = intermittent pneumatic compression; LMWH = low-molecular-weight heparin; PE = pulmonary embolism; VTE = venous thromboembolism.

SUBMITTED February 28, 2017. ACCEPTED September 25, 2017.

INCLUDE WHEN CITING Published online March 30, 2018; DOI: 10.3171/2017.9.JNS17533. 


\section{- CLASSIFICATION OF EVIDENCE}

Type of Question Therapeutic

Study Design Randomized controlled trial

Evidence Class I

\section{Summary Statement}

This underpowered class I study suggests that the addition of intraoperative intermittent pneumatic compression (IPC) reduces the risk of venous thromboembolism (VTE) in patients undergoing intracranial surgery, but the difference in outcome did not reach statistical significance after adjustment for differences between treatment groups.

\section{Classification of Evidence}

Prell and colleagues deliver a rare commodity to the neurosurgical literature: a class I randomized controlled trial. Although one might legitimately question several potential threats to the reliability of this study (e.g., $13 \%$ of randomized patients were not ultimately analyzed, and the majority were apparently lost from the intervention group), these do not warrant downgrading the level of evidence. The authors found that $26.4 \%$ of control patients developed a VTE. That number was reduced to $7.3 \%$ in the IPC treatment arm. This translates into a statistically significant risk difference of $19.1 \%(95 \% \mathrm{Cl}$ $5.9 \%-62.1 \%$ ) and an astoundingly good number needed to treat (5.24). Unfortunately, despite randomization, one important difference between treatment groups persisted: duration of surgery (a known risk factor for VTE) was 255 minutes in the IPC group and 288 minutes in the control arm. The fact that the $p$ value for this difference is 0.11 is unimportant and misleading. The $p$ values only convey information about random error-how likely is the observed difference in duration due to chance. Because this is a randomized trial, this difference must be due to chance. The only relevant question is whether the difference is clinically important. To remedy this imbalance between treatment groups in a potentially important prognostic characteristic, the authors appropriately use a multivariate analytic technique (in this case, multivariate logistic regression). When they do so, the $p$ value for the difference between treatment arms, which was 0.029 in the univariate analysis, becomes 0.069 in the multivariate analysis-no longer statistically significant. This does not mean that the trial is unimportant, and the reason for this failure to achieve statistical significance is instructive. A distressingly rare and important feature of this trial is an a priori sample size calculation. As often happens, however, the assumptions for that calculation (in this case, a $40.6 \%$ baseline risk of VTE and a $60 \%$ risk reduction to $16.2 \%$ ) were not observed in the actual trial. The observed incidences were much lower. As a result, the trial enrolled too few patients to show a statistically significant difference in outcomes between treatment groups, even if a difference truly exists. Statisticians call this a type II error and would say that this trial is underpowered to show the a priori target reduction in VTE (from $40.6 \%$ to $16.2 \%$ ). This does not mean that the trial is unimportant. These results certainly suggest that IPC is a potentially important intervention. They also provide a strong rationale for performing a larger follow-up trial and provide improved information upon which to base a sample size calculation for that larger trial.
Finally, given the lack of observed toxicity and minimal cost from IPC, clinicians who require guidance about how to manage their patients today might reasonably consider adopting this intervention while awaiting the results of the essential confirmatory trial. Even better, they should contribute patients to that new trial.

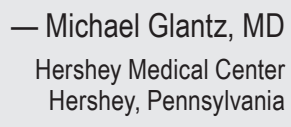

esized that VTE develops during surgery. This is probably due to venous stasis in an immobilized patient in combination with the release of thrombogenic substances from cerebral parenchyma. ${ }^{18,36}$ As a consequence, prevention of VTE would appear to be most efficient by intraoperative means.

Intermittent pneumatic compression (IPC) of the lower extremity is known to be effective in prophylaxis of VTE in immobilized patients (e.g., on ICU wards) because it mimics physiological compression of lower-extremity veins by muscle contraction. ${ }^{6,19,32}$ The superiority of IPC over simple compression stockings has not been demonstrated yet. ${ }^{3}$ However, consistent pre- and postoperative use of IPC was shown to lower the incidence of VTE in patients undergoing orthopedic surgery. ${ }^{33}$ Likewise, its combined intra- and postoperative use seemed to have the same effect in neurosurgical populations in retrospective analysis. ${ }^{14}$

In the present study, we prospectively scrutinized the potential of IPC to prevent DVT (and thus VTE) in neurosurgical patients. The presence or absence of DVT was determined by Doppler sonography of the lower extremity pre- and postoperatively. Hypothesizing intraoperative development of VTE, IPC was exclusively used intraoperatively in the treatment group. The primary end point of this study was the incidence of postoperative VTE with respect to IPC. As a secondary end point, the influence of duration of surgery was to be determined.

\section{Methods \\ Patient Population}

From March 2013 until April 2015, a single-center controlled randomized prospective study was conducted after approval by the university's ethics committee. A total of 108 consecutive patients were initially included; they gave informed consent and were randomized into the treatment group with IPC administration or the control group without IPC. The initial sample size was calculated based on a VTE incidence of $40.6 \%$ observed in a previous study ${ }^{29}$ and a published risk reduction regarding VTE in the range of $60 \%$ for postoperative administration of IPC. ${ }^{35}$ This calculation was performed by chi-square test, assuming a significance of $5 \%$ and a power of $80 \%$.

Undergoing elective surgery for intracranial pathology via craniotomy served as the inclusion criterion. Exclusion criteria were gravidity, age $<18$ years, perioperative administration of blood products, abnormal blood coagu- 
TABLE 1. Clinical variables and possible differences between the IPC and control group

\begin{tabular}{lccc}
\hline \multicolumn{1}{c}{ Variable } & IPC Group & Control Group & p Value \\
\hline No. of patients & 41 & 53 & \\
\hline Mean age in yrs \pm SD & $54.2 \pm 10.9$ & $55.8 \pm 15$ & 0.69 \\
\hline Male/female (\% female) & $17: 24(58.5)$ & $25: 28(52.8)$ & 0.29 \\
\hline Mean BMI \pm SD & $26.36 \pm 4.6$ & $26.94 \pm 5.12$ & 0.57 \\
\hline $\begin{array}{l}\text { Mean duration of surgery in } \\
\text { min } \pm \text { SD }\end{array}$ & $255 \pm 73$ & $288 \pm 121$ & 0.11 \\
\hline Histopathology & & & \\
\hline$\quad$ Meningioma & $10 / 41$ & $9 / 53$ & 0.334 \\
\hline Schwannoma & $15 / 41$ & $21 / 53$ & 0.768 \\
\hline$\quad$ Glioma WHO grades III-IV & $9 / 41$ & $12 / 53$ & 0.909 \\
\hline$\quad$ Glioma WHO grades I-II & $1 / 41$ & $3 / 53$ & 0.343 \\
\hline Metastasis & $2 / 41$ & $3 / 53$ & 0.834 \\
\hline Other & $4 / 41$ & $5 / 53$ & 0.870 \\
\hline
\end{tabular}

$\mathrm{BMI}=$ body mass index .

Statistical significance was not reached.

lation (thrombocytes and/or plasmatic coagulation, antiplatelet agents, preoperative C-reactive protein $>5 \mathrm{mg} / \mathrm{L}$, liver disease), preoperative detection of DVT, varicosis, posthrombotic syndrome, peripheral artery occlusive disease, and unavailability of either pre- or postoperative Doppler sonography. Randomization was carried out externally by the Coordination Center for Clinical Studies Halle (KKSH), which also conducted random sample monitoring. The study was registered in the German Clinical Trials Register (https://www.drks.de), and its registration no. is DRKS00011783.

Fourteen patients were excluded after randomization. This was due to the lack of pre- or postoperative Doppler sonography in 10 patients. In 1 patient with an incidental intracranial aneurysm, the treatment modality was changed from clipping to coiling on short notice. One patient was excluded because the IPC sleeves had been mistakenly attached too late (after dura mater opening). One patient died before postoperative Doppler sonography because of an intraoperative complication unrelated to VTE, and 1 patient had been accidentally included twice.

Of the remaining 94 patients, 52 were female and 42 were male. The mean age was $55 \pm 13.8$ years (mean \pm $\mathrm{SD}$ ), ranging from 21 to 83 years. The mean body mass index was 26.7. A total of $26.6 \%$ of the patients harbored gliomas (WHO grades I-IV). Meningiomas were diagnosed in $20.2 \%$. Metastasis was seen in $5.3 \%$ and vestibular schwannoma in $38.3 \%$. The remaining patients harbored cavernomas $(n=3)$, incidental aneurysms $(n=3)$, aneurysmal bone cyst $(\mathrm{n}=1)$, and trigeminal neuralgia ( $\mathrm{n}$ $=2$ ). Table 1 summarizes all clinical variables.

All patients (in both the treatment and control groups) were treated with compression stockings starting from positioning for surgery and continuing until the 5th postoperative day, combined with subcutaneous administration of LMWH (3000 IU, certoparin), which was started on the 1st postoperative day and continued until discharge. When VTE was diagnosed, therapeutic anticoagulation

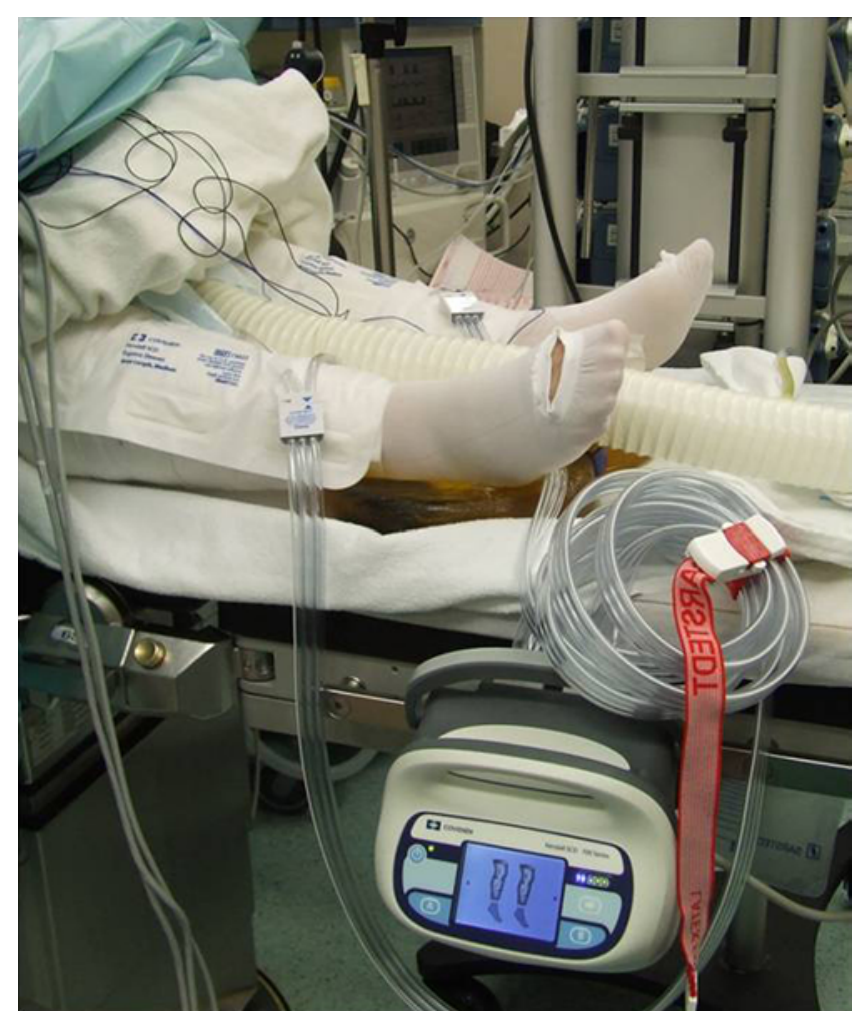

FIG. 1. Administration of IPC sleeves. Calf-length sleeves were used in all patients. The picture shows the operative setup and the air pulse generator. Figure is available in color online only.

was used, with administration of LMWH twice a day at a dosage depending on patient weight and the surgeon's discretion.

\section{Intraoperative IPC Administration}

After induction of anesthesia and before patient positioning, standard calf-length IPC sleeves (multichambered) were applied on top of the regular compression stockings (Fig. 1). Throughout the complete procedure until extubation, these were intermittently inflated to a maximum pressure of $45 \mathrm{~mm} \mathrm{Hg}$ in standard thromboprophylaxis mode by a standard air pulse generator: sequential compression (inflating distal chambers first, then continuing to the more proximal chambers in a wavelike, "milking" fashion) was used once every minute.

\section{Doppler Sonography and Diagnosis of PE}

All examinations were conducted with the same device (Sonoline Antares; Siemens Sector Healthcare) and by the same examiner (B.M.T., who has a level 3 certificate for vascular ultrasound from the German Society for Ultrasound in Medicine [www.degum.de] and who was blinded to the group status of all patients).

Examinations took place on the day before surgery and between the 3rd and 6th day postoperatively. Diagnosis of DVT was based on direct thrombus detection by incomplete compressibility of the vessel and/or observation of absent blood flow. All anatomical levels of the lower-ex- 
tremity venous system were thoroughly examined on both sides, including veins of the pelvis (A-sounds). Diagnosed DVTs were subdivided according to consensus terminology $y^{5}$ into 2 subtypes: 1) proximal DVT either at the level of or cranial to the trifurcation of the popliteal vein; and 2) DVT distal to this level in the deep veins of the lower leg or in muscle veins within the musculus triceps surae.

\section{Statistical Analysis}

Statistical analysis was done with IBM SPSS Statistics 23 software (IBM Corp.). The incidence of VTE in the treatment and control groups was evaluated using Fisher's exact test. Possible differences between the IPC and control groups regarding the pathology operated on were analyzed with the chi-square test of proportions.

Duration of surgery had been confirmed to be an independent risk factor for VTE in a previous study. ${ }^{29}$ Thus, it was compared between both groups (t-test), and its influence on outcome measures was quantified by logistic regression. The latter was also used to determine the influence of IPC administration on the risk of VTE.

\section{Results}

Complications related to IPC use were not observed in this study. Its administration did not interfere with or prolong surgery.

\section{Incidence of VTE}

In the control group, 14 of 53 patients (26.4\%) developed VTE. Six of these lesions $(42.9 \%)$ were located proximally. In contrast, only 3 of 41 patients $(7.3 \%)$ in the treatment group developed VTE, and none of these findings were located proximally (Fig. 2). The difference in VTE incidence between both groups as determined by Fisher's test was significant ( $\mathrm{p}=0.029$ ).

In the control group, 9 of 14 patients with VTE harbored thrombosis in both legs, whereas in the treatment group this was the case in 1 of 3 patients. To reflect this observation, a calculation was done for both legs of each patient separately, thus increasing the sample size from 94 to 188 . In doing so, the incidence of VTE in the control group was found to be $21.7 \%$, whereas it was $4.9 \%$ in the treatment group ( $\mathrm{p}=0.0013)$ (Fig. 3).

In logistic regression analysis, the influence of IPC on the incidence of VTE was statistically significant $(\mathrm{p}=$ 0.016) when each leg was accounted for separately, but did not fully reach significance $(p=0.069)$ when this was not done. Application of IPC led to a risk reduction regarding VTE to roughly one-quarter with both calculations: the factor was 0.28 with both legs and 0.25 with each leg accounted for separately.

\section{Duration of Surgery and Its Influence on VTE}

The mean duration of surgery was 274 minutes, but it differed widely (range 68-620 minutes, SD 103 minutes). There was no significant difference in duration of surgery between the treatment and control groups, as determined by the t-test $(\mathrm{p}=0.11)$.

In logistic regression, the influence of duration of sur-

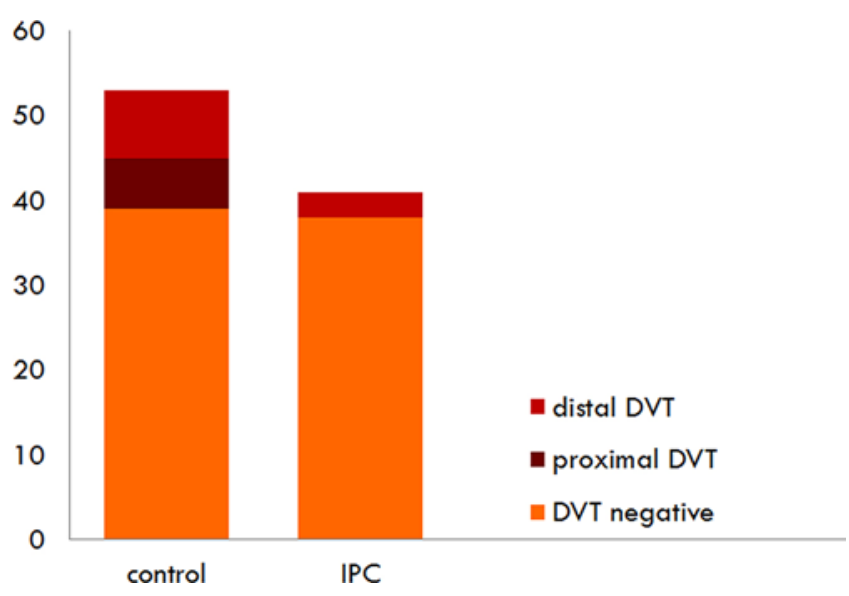

FIG. 2. Bar graph showing incidence of proximal and distal DVT. The figure shows the difference between the IPC and control groups; the incidence of DVT was significantly higher in the control group. No proximal DVT was observed in the IPC group. Values on the y-axis denote percentages. Figure is available in color online only.

gery on VTE incidence was significant $(\mathrm{p}<0.01)$ and highly significant $(\mathrm{p}<0.001)$ when each leg was addressed separately: each hour of surgery would increase the risk of VTE by a factor of 1.56 (no difference between each leg separately or combined).

\section{Discussion}

\section{Findings and Limitations}

The presented data support the hypothesis that intraoperative use of IPC significantly lowers the risk of postoperative VTE in a neurosurgical population. All in all, the risk was approximately quartered by this method in the present study. Control and treatment groups did not differ in a significant way in any of the preoperative clinical variables. However, there was a difference regarding duration of surgery, which did not reach significance, but approximated a trend in terms of statistics $(p=0.11$ after t-test) toward the patients in the IPC group having shorter surgeries. This may influence results because duration of surgery was confirmed to be a risk factor for VTE in this study. However, this influence was statistically eliminated by logistic regression analysis, which showed IPC to be an independent risk factor when each leg was taken into account separately $(\mathrm{p}=0.016)$. When this was not done, significance was not reached $(\mathrm{p}=0.069)$. We believe that this is an effect of sample size, which is artificially raised by analyzing each leg separately. Studies with a higher number of patients may very well show a stronger significance in this regard. A similar effect was seen with Fisher's test: the incidence of VTE was significantly lower in the IPC group $(p=0.029)$, and when each leg was accounted for separately, the significance was improved $(p=0.0013)$.

We insisted that all Doppler examinations of the patients in our cohort were to be performed by the highly qualified head of our angiology department (B.M.T.), and therefore 10 patients had to be excluded because in their cases this specific investigator was found to be unavailable on short notice. This fact additionally influenced the num- 


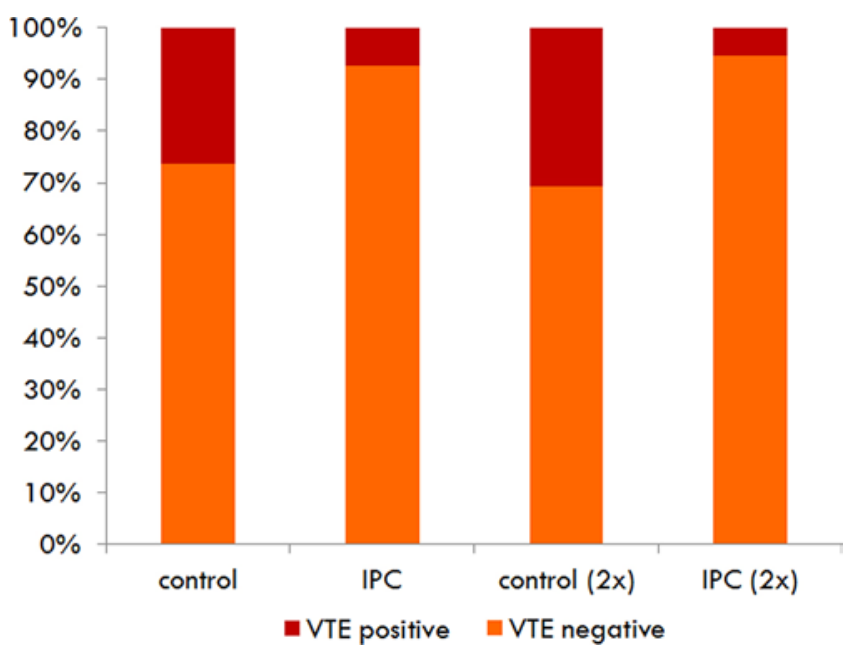

FIG. 3. Bar graph showing VTE incidence. This figure shows the incidence of VTE for the IPC and control groups in general (the 2 left-hand bars) and with each leg taken into account separately (the 2 right-hand bars). According to the latter calculation, the difference between both groups reached high significance $(p<0.001)$. Figure is available in color online only.

ber of patients eligible for final data analysis in a negative way. However, the results were found to be significant despite these limitations.

Last, an unusually large percentage of our patients $(38.3 \%)$ were surgically treated for vestibular schwannoma. This deviation from a standard neurosurgical patient population may also influence transferability to the situation in other institutions.

\section{The Impact of IPC on VTE Incidence as Reflected by the Literature}

Virchow stated as early as 1846 his "triad" of conditions that may lead to VTE:4 1) stasis; 2) hypercoagulability; and 3) endothelial injuries.

Although it is known that malignant disease is associated with an increased risk of VTE, ${ }^{27,28}$ brain surgery in itself seems to imply additional risks. ${ }^{36}$ In neurosurgical patients, hypercoagulability may be attributed to manipulation of brain tissue, which contains high concentrations of thromboplastin. ${ }^{18}$ According to Heesen et al., the plasma concentration of the thrombin-antithrombin III complex will reach its peak 3 hours after the beginning of surgery.

As complex intracranial surgery may take many hours to complete, this peak will be reached almost routinely. In addition, patients are immobile for the whole duration of surgery, which implies venous stasis. Given the high incidence of VTE in neurosurgical patients, ${ }^{11,17}$ it appears highly plausible that many of these postoperative VTEs will develop not after but during surgery itself. Reflecting this, a highly significant correlation between duration of surgery and the incidence of VTE had been demonstrated before. ${ }^{29}$ In the present study, this correlation was confirmed. Each hour of surgery will increase the risk of postoperative VTE by a factor of 1.56 in logistic regression analysis.

Duration of surgery cannot always be reduced in response to these findings; thus, risk management is bound to take different paths. We believe that the key to this is venous stasis, which may be influenced by comparatively simple means. With IPC of the lower extremity, the work of the skeletal muscle pump can be simulated, ${ }^{6}$ which will improve venous blood flow to the heart from the lower extremity ${ }^{3,32}$ and thus reduce venous stasis. In addition, IPC seems to increase fibrinolysis, which may contribute to the reduction of VTE risk. ${ }^{8,19}$ Surprisingly, despite these consistent findings, the superiority of intraoperative IPC over standard compression stockings has not been proven yet. ${ }^{3}$

The highest-quality data available in the literature have been acquired from orthopedic patients. Based on $>2400$ patients pooled from several surgical studies, the evidence-based clinical practical guidelines on VTE prevention in patients undergoing orthopedic surgery ${ }^{13}$ state that IPC seems to have an "apparently large effect" when added to chemoprophylaxis; however, because of methodological limitations of the included studies, the guidelines advise careful interpretation of the data. In their conclusions, they recommend perioperative dual use of IPC and chemoprophylaxis in patients undergoing major orthopedic surgery, even though the evidence level is considered to be no more than class IIc.

A Cochrane review of the surgical literature as a whole identified only 11 studies of sufficiently high quality for further analysis. ${ }^{20}$ Six of these had been performed on patients who underwent orthopedic surgery; the remaining 5 studies dealt with patients undergoing urological, cardiothoracic, general surgery, and gynecological procedures. In this review, the authors concluded that IPC lowers the incidence of DVT in a significant way. They recommended its use especially in high-risk patients, and stated that more studies on this topic are urgently needed.

The Cochrane review was updated in 2016, ${ }^{21}$ and 22 trials were included then. Studies in which IPC had only been used for a very short time (e.g., only intraoperatively, as in the present study) were systematically excluded from the review. In the present study, however, we were able to show that the positive effects of IPC are obviously available with intraoperative use only. This is important because compliance has been considered to be a major problem when patients were expected to wear IPC devices for several days postoperatively. ${ }^{9,33}$ Because of this important difference, the results and conclusions from the review may be only partially transferable to neurosurgical patients treated with IPC exclusively intraoperatively. On the basis of 11 of the 22 trials, the authors of the updated Cochrane review stated that IPC in combination with medication influences the incidence of $\mathrm{PE}$ in a positive way, but does not lower the incidence of DVT as compared with medication alone. Nevertheless, they recommended the combined use of IPC with medication despite "weak" to "moderate" quality evidence. The downgrade of evidence quality was due to the risk of bias and imprecision within the included studies.

Only 1 of the studies included in the Cochrane review actually dealt with neurosurgical patients. In this particular trial, IPC was not found to be superior to medication or combined therapy. The study was terminated due to adverse effects of enoxaparin initiated at the time of anesthesia. $^{12}$ 
Therefore, strictly speaking, there is as yet only a weak evidence base for use of IPC in patients undergoing neurosurgery. Available data that are supportive for IPC in patients who have undergone craniotomy are retrospective by nature, ${ }^{14}$ and there also exist prospective data obtained in neurosurgical patients that could not demonstrate the superiority of IPC over chemoprophylaxis alone or conventional compression stockings. ${ }^{3,12,24}$ Nevertheless, despite this equivocality, the data taken from other surgical subspecialties have obviously convinced practitioners in many neurosurgical departments to use IPC. This is especially true in the US; in a contemporary review of VTE prophylaxis in neurosurgery, the authors state that IPC was used as the main means for mechanical VTE prophylaxis in all US centers that contributed data to the review. ${ }^{2}$ However, in their conclusion they ultimately chose to recommend mechanical prophylaxis (along with unfractionated heparin) without further differentiation between IPC and compression stockings: as yet, there is no proven superiority of IPC over compression stockings in patients undergoing neurosurgery. ${ }^{3}$

In the present study, such superiority has now been demonstrated by a controlled, prospective, and randomized single-center setup. The incidence of VTE was roughly quartered after application of IPC. Moreover, proximal DVT, which has been shown to carry a significantly larger risk of potentially fatal PE, ${ }^{15,34}$ was not seen at all in the treatment group. Strictly intraoperative use of IPC was sufficient to evoke this effect.

\section{Conclusions}

Intraoperative use of IPC quarters the risk of VTE in patients undergoing craniotomy. Because hypercoagulability and venous stasis are known risk factors for VTE, and because the risk of VTE is correlated with the duration of surgery, this effect is most probably caused by improved blood flow in the lower extremity. As a consequence drawn from the data shown, intraoperative use of IPC has become routine in our institution in cranial surgery.

Future studies will hopefully confirm our observations with a higher number of patients, which would best be achievable in a multicenter setup.

\section{Acknowledgments}

Funding was received from the Wilhelm Roux Program of the Martin Luther University of Halle-Wittenberg, Germany (FKZ 26/02).

\section{References}

1. Agnelli G, Piovella F, Buoncristiani P, Severi P, Pini M, D’Angelo A, et al: Enoxaparin plus compression stockings compared with compression stockings alone in the prevention of venous thromboembolism after elective neurosurgery. $\mathbf{N}$ Engl J Med 339:80-85, 1998

2. Algattas H, Damania D, DeAndrea-Lazarus I, Kimmell KT, Marko NF, Walter KA, et al: Systematic review of safety and cost-effectiveness of venous thromboembolism prophylaxis strategies in patients undergoing craniotomy for brain tumor. Neurosurgery [epub ahead of print], 2017

3. Auguste KI, Quiñones-Hinojosa A, Berger MS: Efficacy of mechanical prophylaxis for venous thromboembolism in patients with brain tumors. Neurosurg Focus 17(4):E3, 2004

4. Bagot CN, Arya R: Virchow and his triad: a question of attribution. Br J Haematol 143:180-190, 2008

5. Caggiati A, Bergan JJ, Gloviczki P, Eklof B, Allegra C, Partsch H: Nomenclature of the veins of the lower limb: extensions, refinements, and clinical application. J Vasc Surg 41:719-724, 2005

6. Calnan JS, Pflug JJ, Mills CJ: Pneumatic intermittentcompression legging simulating calf-muscle pump. Lancet 2:502-503, 1970

7. Chan AT, Atiemo A, Diran LK, Licholai GP, McLaren Black $\mathrm{P}$, Creager MA, et al: Venous thromboembolism occurs frequently in patients undergoing brain tumor surgery despite prophylaxis. J Thromb Thrombolysis 8:139-142, 1999

8. Comerota AJ, Chouhan V, Harada RN, Sun L, Hosking J, Veermansunemi R, et al: The fibrinolytic effects of intermittent pneumatic compression: mechanism of enhanced fibrinolysis. Ann Surg 226:306-314, 1997

9. Comerota AJ, Katz ML, White JV: Why does prophylaxis with external pneumatic compression for deep vein thrombosis fail? Am J Surg 164:265-268, 1992

10. Danish SF, Burnett MG, Stein SC: Prophylaxis for deep venous thrombosis in patients with craniotomies: a review. Neurosurg Focus 17(4):E2, 2004

11. Dermody M, Alessi-Chinetti J, Iafrati MD, Estes JM: The utility of screening for deep venous thrombosis in asymptomatic, non-ambulatory neurosurgical patients. J Vasc Surg 53:1309-1315, 2011

12. Dickinson LD, Miller LD, Patel CP, Gupta SK: Enoxaparin increases the incidence of postoperative intracranial hemorrhage when initiated preoperatively for deep venous thrombosis prophylaxis in patients with brain tumors. Neurosurgery 43:1074-1081, 1998

13. Falck-Ytter Y, Francis CW, Johanson NA, Curley C, Dahl OE, Schulman S, et al: Prevention of VTE in orthopedic surgery patients: Antithrombotic Therapy and Prevention of Thrombosis, 9th ed: American College of Chest Physicians Evidence-Based Clinical Practice Guidelines. Chest 141 (2 Suppl):e278S-e325S, 2012

14. Frisius J, Ebeling M, Karst M, Fahlbusch R, Schedel I, Gerganov V, et al: Prevention of venous thromboembolic complications with and without intermittent pneumatic compression in neurosurgical cranial procedures using intraoperative magnetic resonance imaging. A retrospective analysis. Clin Neurol Neurosurg 133:46-54, 2015

15. Galanaud JP, Quenet S, Rivron-Guillot K, Quere I, Sanchez Muñoz-Torrero JF, Tolosa C, et al: Comparison of the clinical history of symptomatic isolated distal deep-vein thrombosis vs. proximal deep vein thrombosis in 11086 patients. J Thromb Haemost 7:2028-2034, 2009

16. Gillet JL, Perrin MR, Allaert FA: Short-term and mid-term outcome of isolated symptomatic muscular calf vein thrombosis. J Vasc Surg 46:513-519, 2007

17. Hamilton MG, Hull RD, Pineo GF: Venous thromboembolism in neurosurgery and neurology patients: a review. Neurosurgery 34:280-296, 1994

18. Heesen M, Kemkes-Matthes B, Deinsberger W, Boldt J, Matthes KJ: Coagulation alterations in patients undergoing elective craniotomy. Surg Neurol 47:35-38, 1997

19. Jacobs DG, Piotrowski JJ, Hoppensteadt DA, Salvator AE, Fareed J: Hemodynamic and fibrinolytic consequences of intermittent pneumatic compression: preliminary results. $\mathbf{J}$ Trauma 40:710-717, 1996

20. Kakkos SK, Caprini JA, Geroulakos G, Nicolaides AN, Stansby G, Reddy DJ: Combined intermittent pneumatic leg compression and pharmacological prophylaxis for prevention of venous thromboembolism in high-risk patients. Cochrane Database Syst Rev 4:CD005258, 2008 
21. Kakkos SK, Caprini JA, Geroulakos G, Nicolaides AN, Stansby G, Reddy DJ, et al: Combined intermittent pneumatic leg compression and pharmacological prophylaxis for prevention of venous thromboembolism. Cochrane Database Syst Rev 9:CD005258, 2016

22. Kearon C: Natural history of venous thromboembolism. Circulation 107 (23 Suppl 1):I22-I30, 2003

23. Khaldi A, Helo N, Schneck MJ, Origitano TC: Venous thromboembolism: deep venous thrombosis and pulmonary embolism in a neurosurgical population. J Neurosurg 114:40-46, 2011

24. Kurtoglu M, Yanar H, Bilsel Y, Guloglu R, Kizilirmak S, Buyukkurt D, et al: Venous thromboembolism prophylaxis after head and spinal trauma: intermittent pneumatic compression devices versus low molecular weight heparin. World J Surg 28:807-811, 2004

25. Masuda EM, Kistner RL: The case for managing calf vein thrombi with duplex surveillance and selective anticoagulation. Dis Mon 56:601-613, 2010

26. Palareti G, Cosmi B, Lessiani G, Rodorigo G, Guazzaloca G, Brusi C, et al: Evolution of untreated calf deep-vein thrombosis in high risk symptomatic outpatients: the blind, prospective CALTHRO study. Thromb Haemost 104:1063-1070, 2010

27. Prandoni P, Falanga A, Piccioli A: Cancer and venous thromboembolism. Lancet Oncol 6:401-410, 2005

28. Prandoni P, Piccioli A, Girolami A: Cancer and venous thromboembolism: an overview. Haematologica 84:437445, 1999

29. Prell J, Rachinger J, Smaczny R, Taute BM, Rampp S, Illert $\mathrm{J}$, et al: D-dimer plasma level: a reliable marker for venous thromboembolism after elective craniotomy. J Neurosurg 119:1340-1346, 2013

30. Rachinger JC, Koman G, Scheller C, Prell J, Rampp S, Strauss C: Practice in the perioperative prevention of deep vein thrombosis in German neurosurgical departments: is there a trend towards homogenization? Cent Eur Neurosurg 72:115-119, 2011

31. Righini M, Paris S, Le Gal G, Laroche JP, Perrier A, Bounameaux H: Clinical relevance of distal deep vein thrombosis. Review of literature data. Thromb Haemost 95:56-64, 2006

32. Roberts VC, Sabri S, Beeley AH, Cotton LT: The effect of intermittently applied external pressure on the haemodynamics of the lower limb in man. Br J Surg 59:223-226, 1972

33. Silbersack Y, Taute BM, Hein W, Podhaisky H: Prevention of deep-vein thrombosis after total hip and knee replacement. Low-molecular-weight heparin in combination with intermittent pneumatic compression. J Bone Joint Surg Br 86:809-812, 2004

34. Stein PD, Matta F, Musani MH, Diaczok B: Silent pulmonary embolism in patients with deep venous thrombosis: a systematic review. Am J Med 123:426-431, 2010

35. Urbankova J, Quiroz R, Kucher N, Goldhaber SZ: Intermittent pneumatic compression and deep vein thrombosis prevention. A meta-analysis in postoperative patients. Thromb Haemost 94:1181-1185, 2005

36. Vukovich TC, Gabriel A, Schaeffer B, Veitl M, Matula C, Spiss CK: Hemostasis activation in patients undergoing brain tumor surgery. J Neurosurg 87:508-511, 1997

37. White RH, Zhou H, Romano PS: Incidence of symptomatic venous thromboembolism after different elective or urgent surgical procedures. Thromb Haemost 90:446-455, 2003

\section{Disclosures}

The authors report no conflict of interest concerning the materials or methods used in this study or the findings specified in this paper.

\section{Author Contributions}

Conception and design: Prell, Marquart, Strauss. Acquisition of data: Schenk, Taute, Marquart. Analysis and interpretation of data: Prell, Schenk, Rampp. Drafting the article: Prell. Critically revising the article: Schenk, Scheller, Strauss, Rampp. Reviewed submitted version of manuscript: Schenk, Taute, Scheller, Marquart, Strauss, Rampp. Approved the final version of the manuscript on behalf of all authors: Prell. Statistical analysis: Prell, Schenk, Rampp. Administrative/technical/material support: Taute, Scheller, Marquart, Strauss. Study supervision: Prell.

\section{Correspondence}

Julian Prell: University of Halle, Germany. julian.prell@medizin. uni-halle.de. 